\title{
Evaluation of the imputation performance of the program IMPUTE in an admixed sample from Mexico City using several model designs
}

S Krithika', Adán Valladares-Salgado ${ }^{2}$, Jesus Peralta², Jorge Escobedo-de La Peña ${ }^{3}$, Jesus Kumate-Rodríguez ${ }^{4}$, Miguel Cruz $^{2}$ and Esteban J Parra ${ }^{1 *}$

\begin{abstract}
Background: We explored the imputation performance of the program IMPUTE in an admixed sample from Mexico City. The following issues were evaluated: (a) the impact of different reference panels (HapMap vs. 1000 Genomes) on imputation; (b) potential differences in imputation performance between single-step vs. two-step (phasing and imputation) approaches; (c) the effect of different posterior genotype probability thresholds on imputation performance and (d) imputation performance in common vs. rare markers.

Methods: The sample from Mexico City comprised 1,310 individuals genotyped with the Affymetrix 5.0 array. We randomly masked $5 \%$ of the markers directly genotyped on chromosome $12(n=1,046)$ and compared the imputed genotypes with the microarray genotype calls. Imputation was carried out with the program IMPUTE. The concordance rates between the imputed and observed genotypes were used as a measure of imputation accuracy and the proportion of non-missing genotypes as a measure of imputation efficacy.

Results: The single-step imputation approach produced slightly higher concordance rates than the two-step strategy ( $99.1 \%$ vs. $98.4 \%$ when using the HapMap phase II combined panel), but at the expense of a lower proportion of non-missing genotypes (85.5\% vs. 90.1\%). The 1,000 Genomes reference sample produced similar concordance rates to the HapMap phase II panel (98.4\% for both datasets, using the two-step strategy). However, the 1000 Genomes reference sample increased substantially the proportion of non-missing genotypes ( $94.7 \%$ vs. 90.1\%). Rare variants (<1\%) had lower imputation accuracy and efficacy than common markers.
\end{abstract}

Conclusions: The program IMPUTE had an excellent imputation performance for common alleles in an admixed sample from Mexico City, which has primarily Native American (62\%) and European (33\%) contributions. Genotype concordances were higher than $98.4 \%$ using all the imputation strategies, in spite of the fact that no Native American samples are present in the HapMap and 1000 Genomes reference panels. The best balance of imputation accuracy and efficiency was obtained with the 1,000 Genomes panel. Rare variants were not captured effectively by any of the available panels, emphasizing the need to be cautious in the interpretation of association results for imputed rare variants.

\section{Background}

Genome-wide association studies (GWAS) are a convenient and powerful tool for the identification of common genetic variants associated with complex diseases [1-5]. In recent years, high-density GWAS have proven successful in identifying loci predisposing to a variety of complex diseases, e.g., type 1 and type 2 diabetes,

\footnotetext{
*Correspondence: esteban.parra@utoronto.ca

'Department of Anthropology, University of Toronto at Mississauga, 3359

Mississauga Road North, Mississauga, ON, Canada

Full list of author information is available at the end of the article
}

obesity, inflammatory bowel disease, prostate cancer and breast cancer $[5,6]$. The recent successes of GWAS have mainly been possible due to the rapid advancement in high-throughput SNP genotyping technologies (e.g., Affymetrix and Illumina platforms), which assay a large number of SNPs (between 100,000 and 2,500,000) across the human genome [7-9]. However, despite recent improvements, the coverage of most of the genotyping platforms remains relatively inadequate, in comparison with the total number of SNPs described in the genome. Furthermore, rare variants are typically not included in 
these genotyping arrays and a fraction of the typed SNPs are eliminated from further analyses, due to genotyping problems, leading to the loss of statistical power in association studies [10-13].

To overcome the aforementioned limitations of GWAS genotyping platforms, a variety of imputation methods have been developed. These methods infer missing or untyped SNP genotypes based on the genotypes at nearby typed SNPs, using the pattern of linkage disequilibrium (LD) observed in reference samples. Imputation methods have been extensively used to predict the genotypes of untyped markers by combining reference panels of individuals genotyped at a dense set of SNPs with a study sample genotyped at a subset of the SNPs [14,15]. The main challenge of imputation, however, lies in the selection of an appropriate reference panel relevant for the study populations. Although this is straightforward in samples with ancestry matching that of the available reference panels (e.g., European or East Asian ancestry), this is not the case for samples that are not well represented in the reference panels (e.g. Native American samples or admixed samples). One of the proposed solutions to the latter scenario is to include mixtures of the available reference panels for imputation. It has been described that this strategy results in good imputation accuracy [16].

The application of imputation methods is cost effective, increases the power and coverage of the study, facilitates meta-analysis, enables combination of data across multiple genotyping platforms, and aids in replication of significant findings $[15,17,18]$. Several imputation methods are currently available, based on different statistical models. Commonly used imputation programs are IMPUTE $[19,20]$, MACH [21], BEAGLE [22], fastPHASE [23] and PLINK [24]. The relative performance of these programs has been assessed in various studies [19,25-29].

In the present study, we employed the HapMap and the recently available 1000 Genomes reference panels to evaluate the performance of the imputation program IMPUTE in an admixed sample from Mexico City. The following issues were evaluated in this project: (a) the impact of different reference panels (HapMap and 1000 Genomes) on imputation; (b) potential differences in imputation performance between single-step vs. two-step (phasing and imputation) approaches; (c) the impact of different posterior genotype probability thresholds on imputation performance and (d) imputation performance in common vs. rare markers.

\section{Methods}

\section{Study participants and Genotyping}

A total of 1,310 individuals from Mexico City (967 with type 2 diabetes and 343 with normal glucose tolerance) were analyzed in this study. Informed consent was obtained from each participant, and the research was approved by the ethical research boards of the Medical Center 'Siglo XXI' and the University of Toronto. Genotyping of the sample was then carried out in the microarray analysis facility located in the Centre for Applied Genomics (Toronto, ON, Canada), using the Affymetrix Genome-wide Human SNP array 5.0 (Affymetrix, Santa Clara, CA, USA), and following standard protocols. Further details about participant recruitment and quality control measures can be found elsewhere [30].

\section{Reference panels for imputation}

The following reference panels were used for the present study:

(a) HapMap phase II combined sample, which includes up to 4 million SNPs typed in 269 individuals belonging to East Asian/European/West African ancestry,

(b)HapMap phase II combined sample along with the HapMap phase III Mexican-American LA sample (MXL), which was genotyped for about 1.4 million SNPs, and the

(c) 1000 Genomes phase I sample (June 2011 release), which comprises $>37$ million autosomal SNPs typed in 1,094 individuals from populations around the world (more information is available at http:// www.1000genomes.org/).

\section{Imputation using IMPUTE}

The program IMPUTE v2 $[19,20]$ was employed for imputation of untyped markers using the following strategies: $1 /$ Phasing and imputation in a single analytical step using the HapMap phase II combined reference dataset (singlestep strategy), 2/ Phasing and imputation in a single analytical step using the HapMap phase II combined + HapMap phase III Mexican-American reference datasets (single-step strategy), 3/ Phasing the study sample first and performing imputation using the HapMap Phase II combined reference dataset (two-step strategy), and 4/ Phasing the study sample first and performing imputation using the 1000 Genomes Phase I (June 2011 release) combined reference panel (two-step strategy).

In order to evaluate the performance of the imputation, we randomly masked $5 \%$ of the markers directly genotyped on chromosome $12(\mathrm{n}=1,046)$ and compared the imputed genotypes with the Affymetrix genome-wide Human SNP array 5.0 genotype calls. For the single-step strategy, chromosome 12 was divided into chunks of $15 \mathrm{Mb}$ length (chunk size specified using the -int option). Each chunk was then directly imputed with the following settings: buffer $=250 \mathrm{~kb}, \mathrm{k}=40$, iter $=30$, bur- 
$\operatorname{nin}=10, \mathrm{Ne}=11418$, using the said reference panels. The -buffer option helps to avoid edge effects when imputing in relatively small chunks. For analysis using the twostep strategy, chromosome 12 was broken into smaller chunks of $\sim 5 \mathrm{Mb}$ each, and we also used a buffer region of $250 \mathrm{~kb}$. Phasing of GWAS data in each chunk was subsequently performed to produce the best-guess haplotypes (using -phase and -include_buffer_in_output flags with IMPUTE v2's settings: $k=80$, iter $=30$, bur$\operatorname{nin}=10, \mathrm{Ne}=11500$ ). Imputation from the best-guess haplotypes was then carried out, for each chunk, using the aforementioned reference panels. The differences in imputation settings between the one-step and two-step approaches (e.g. chunk size, k-parameters) are primarily due to the fact that the imputations were done at different times.

\section{Evaluation of imputation performance}

We report the concordance rate between the imputed and observed genotypes for the masked SNPs as a measure of imputation accuracy and the proportion of non-missing genotypes under a given posterior probability threshold as a measure of the imputation efficacy. The program Gtool (http://www.well.ox.ac.uk/ cfreeman/software/gwas/gtool. html) was used for this purpose, using the default posterior probability threshold value of 0.9 to export the IMPUTE data to PLINK format. With this threshold, imputed genotypes with posterior probabilities $<0.9$ were labeled as missing genotypes. Then, the PLINK's -merge command along with -merge-mode 7 command was used to evaluate the genotype concordance. We also used PLINK to obtain information on the proportion of nonmissing genotypes for each of the four imputation strategies.

We also evaluated the imputation performance (accuracy and efficacy) at different posterior probability threshold values $(0.8,0.7,0.6$ and 0.5$)$, in addition to the default threshold value of 0.9. This analysis was carried out only for the two-step imputation method based on the HapMap phase II combined reference sample.
Finally, we explored the effect of allele frequency on imputation performance. The marker INFO scores based on the two-step imputation method using the HapMap phase II combined and the 1000 Genomes (June 2011 release) reference panels were compared for different allele frequency categories, grouping markers in 5\% bins. INFO scores are used to measure imputation quality for each marker, with a value of 1 indicating that there is no uncertainty in the imputed genotypes and a value of 0 indicating that there is complete uncertainty in the genotypes [14]. Histograms were generated to show the distribution of the INFO scores for each bin and the distribution of the differences in INFO scores, and we estimated the correlation between the INFO scores for the two imputation approaches. We also did a more detailed analysis of imputation accuracy and efficacy for markers in the following allele frequency categories: $<1 \%, 1-5 \%$ and $45-50 \%$, using the two-step imputation method and the 1000 Genomes reference panel.

\section{Results}

The concordance rates and the proportion of non-missing genotypes obtained with the four imputation strategies evaluated in this study are shown in Table 1. For this analysis, imputed genotypes with posterior probabilities lower than 0.9 were defined as missing genotypes. The concordance rate was used as a measure of the imputation accuracy and the proportion of non-missing genotypes as a measure of imputation efficacy. The concordance rate was consistently high $(>98 \%)$ for all the imputation strategies, but there were differences between methods in imputation efficacy. Using the single-step strategy produced slightly higher concordance rates than the two-step strategy (e.g. 99.1\% vs. $98.4 \%$ when using the HapMap phase II combined reference sample, respectively), but at the expense of a lower proportion of non-missing genotypes ( $85.5 \%$ vs. $90.1 \%$, respectively). The inclusion of the HapMap phase III Mexican American sample as a reference sample, in addition to the HapMap phase II combined sample, produced a marginal improvement both in concordance rate and proportion of non-

Table 1 Concordance rate and proportion of non-missing genotypes (using a posterior probability threshold of 0.9 ) for chromosome 12 markers in the studied reference panels

\begin{tabular}{|c|c|c|c|}
\hline Reference Panel & IMPUTE Version & $\begin{array}{c}\text { Concordance rate } \\
(\%)\end{array}$ & $\begin{array}{l}\text { Proportion of } \\
\text { non-missing } \\
\text { genotypes (\%) }\end{array}$ \\
\hline $\begin{array}{l}\text { HapMap phase } \\
\text { Il combined }\end{array}$ & Version 2 (Single-step) & 99.09 & 85.5 \\
\hline $\begin{array}{l}\text { HapMap phase II } \\
\text { combined + MXL }\end{array}$ & Version 2 (Single-step) & 99.37 & 85.9 \\
\hline HapMap phase II combined & Version 2 (Two-steps: Phasing \& Imputation) & 98.40 & 90.1 \\
\hline $\begin{array}{l}\text { 1,000 Genomes Phase I } \\
\text { (June } 2011 \text { release) }\end{array}$ & Version 2 (Two-steps: Phasing \& Imputation) & 98.44 & 94.7 \\
\hline
\end{tabular}


missing genotypes $(99.4 \%$ vs. $99.1 \%$ for concordance, and $85.9 \%$ vs. $85.5 \%$ for the proportion of non-missing genotypes, using the single-step approach). For the two-step approach, using the 1,000 Genomes reference sample did not alter the concordance rate with respect to the HapMap phase II combined sample (98.4\% for both datasets). However, the use of the 1000 Genomes panel produced a substantial increase in the proportion of non-missing genotypes ( $94.7 \%$ vs. $90.1 \%$, respectively).

Figure 1 illustrates the concordance rates and the proportion of non-missing genotypes obtained using various posterior probability thresholds. This analysis provides an indication of how the selection of genotype confidence thresholds affects the accuracy and efficacy of the imputations. We restricted this evaluation to the twostep protocol using the HapMap phase II combined sample. As expected, lowering the posterior probability thresholds resulted in progressively reduced concordance rates and higher proportions of non-missing genotypes. Using a threshold of 0.9 , the concordance rate was $98.4 \%$ and the proportion of non-missing genotypes $90.1 \%$. Using a much less conservative threshold of 0.5 , the concordance rate was still quite high (95.5\%) and the proportion of non-missing genotypes went up to $99.6 \%$.

Figure 2 depicts the average marker INFO scores for different allele frequency bins, using the two-step imputation methods based on the HapMap phase II combined and the 1000 Genomes phase I (June 2011 release) reference panels. This figure provides information about imputation quality across the allele frequency spectrum, based on the two reference panels. The average INFO scores obtained for the 1000 Genomes panel are substantially higher, irrespective of the allele frequencies, than the HapMap phase II combined panel. It is also evident in the plot that rare variants (frequencies $<5 \%$ ) have considerably lower INFO scores than common variants. In addition to average imputation qualities, it is also relevant to explore the distribution of INFO scores in each frequency bin. This is depicted in Figures 3A (for the HapMap Phase II combined reference sample) and 3B (for the 1000 Genomes phase I panel). These Figures show that for most frequency bins, the majority of the untyped SNPs have INFO scores higher than 0.9, with decreasing proportions of markers in the lower INFO score categories. However, for rare markers, particularly those with frequencies $<1 \%$, the distribution is considerably wider, and the mode of the distribution does not correspond to the INFO score $>0.9$, but to lower INFO score values. Additionally, the plots also demonstrate that using the 1000 Genomes sample as a reference sample shifts the distributions to the right in all the frequency bin categories. Markers imputed using the 1000 Genomes reference sample tend to have INFO scores higher than those imputed using the HapMap Phase II combined reference panel for all the frequency bins. This is also evident in Figure 4, which shows a histogram showing the distribution of the differences in INFO scores between the two-step imputation methods based on the 1000 Genomes Phase I and the HapMap Phase II combined reference samples. The correlation between the INFO scores of the two-step imputation methods based on the 1000 Genomes Phase I and the HapMap Phase II combined reference samples is shown in Figure 5 . The INFO scores are highly correlated $\left(r^{2}=0.82\right)$ : imputed markers with low INFO scores using the HapMap phase II combined panel also exhibit low INFO scores employing the 1000 Genomes phase I panel, although as described before, markers imputed with the 1000 Genomes panel showed higher INFO scores relative to those imputed with the HapMap sample.

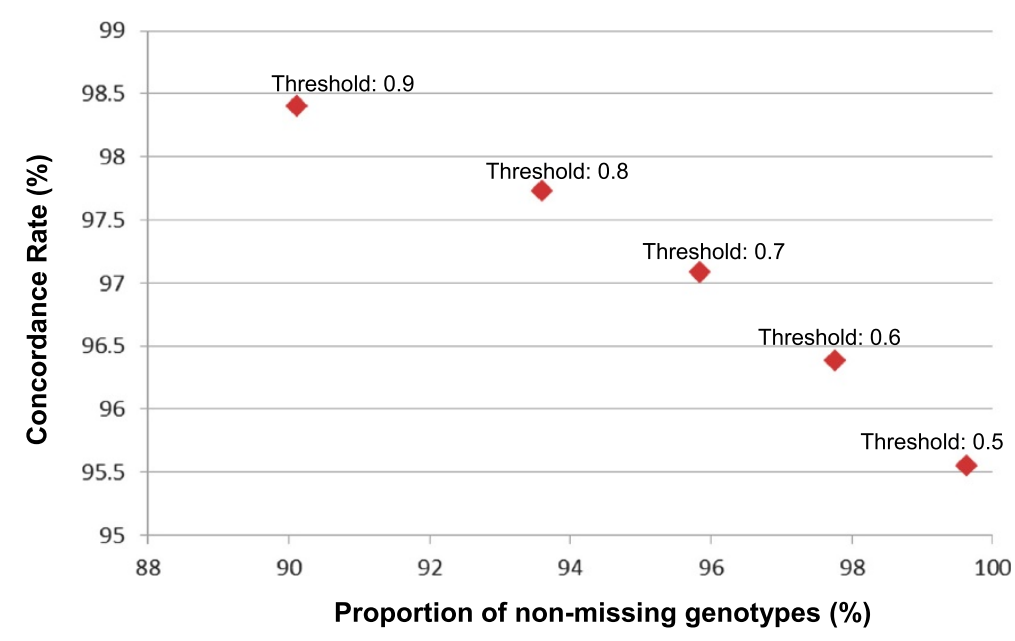

Figure 1 Proportion of non-missing genotypes versus concordance rates using different posterior probability thresholds. This analysis was performed for the HapMap phase II combined reference sample based on the two-step imputation method. 


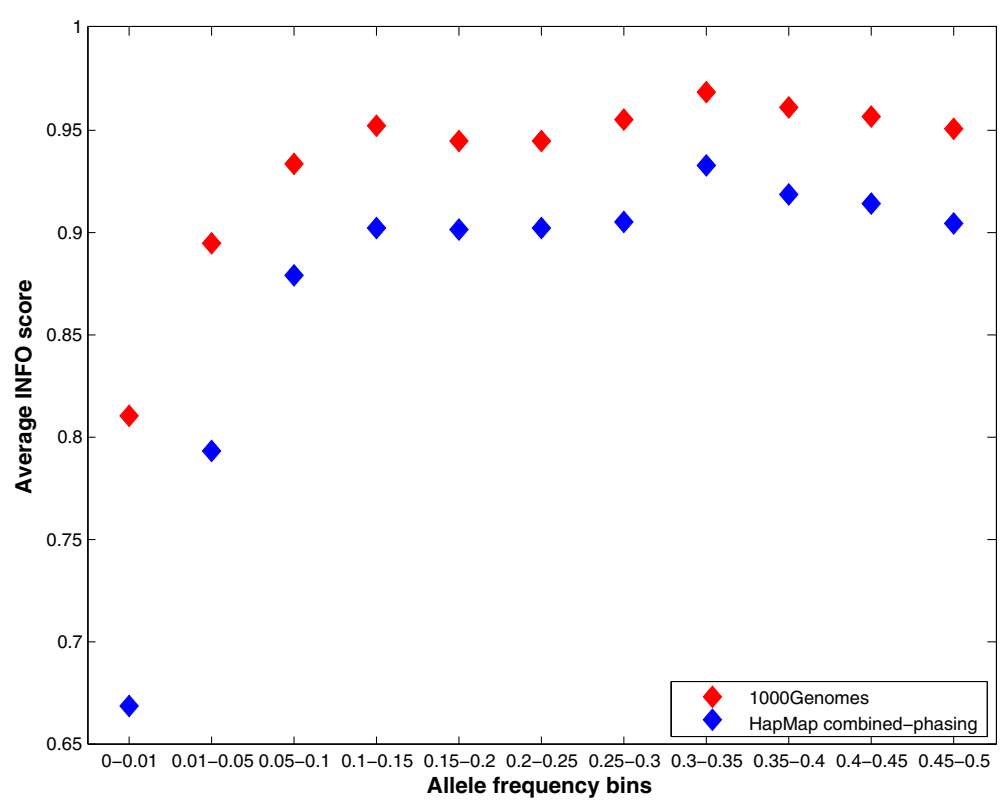

Figure 2 Average INFO scores for different allele frequency bins of the HapMap phase II combined and the 1000 Genomes phase I reference panels. The blue diamond represents the HapMap phase II combined panel and the red diamond represents the 1000 Genomes phase I panel (June 2011 release), based on the two-step imputation method.

We compared in more detail the imputation accuracy and efficacy for markers in the following allele frequency categories: $<1 \%, 1-5 \%$ and $45-50 \%$, using the two-step imputation method and the 1000 Genomes reference panel. For this analysis, instead of using the overall imputation concordance based on the three possible genotypes, we focused our attention on the concordance and missingness rates for the heterozygotes. The reason for employing this strategy is that an analysis based on overall imputation concordance may give misleading results for rare markers: the overall concordance rate may be high for these markers, but the concordance rates for heterozygotes and minor allele homozygotes may be much lower than the overall concordance rates. For the imputed markers in the $45-50 \%$ allele frequency bin, using a posterior probability threshold of 0.9 , the concordance rate for the heterozygotes was $97.5 \%$ and the proportion of non-missing genotypes $90.2 \%$. For the markers in the $1-5 \%$ bin, the concordance rate dropped to $85.4 \%$ and the proportion of non-missing genotypes to $85.1 \%$. For rare markers $(<1 \%)$, the drop was even more pronounced: the concordance rate was only $60.6 \%$ and the proportion of non-missing genotypes was $78.1 \%$.

The results described above are based on markers located on chromosome 12. In order to evaluate the generalizability of these results, we also masked $5 \%$ of genotyped markers on chromosome 22, and on the HLA region, which spans approximately 5 megabases on chromosome 6 and has been under selective pressure in different population groups [31-33]. These analyses were carried out with the two-step imputation method using the HapMap and 1000 Genomes reference panels. For chromosome 22, using the HapMap reference panel, the concordance rate was $97.6 \%$, and the proportion of nonmissing genotypes $83.2 \%$, and using the 1000 Genomes reference panel, the concordance rate was $97.3 \%$ and the proportion of non-missing genotypes $89.9 \%$. For the HLA region, using the HapMap reference panel the concordance rate was $99.35 \%$ and the proportion of nonmissing genotypes $97.4 \%$, and with the 1000 Genomes reference panel the concordance rate was $99.5 \%$ and the proportion of non-missing genotypes $99.05 \%$.

\section{Discussion}

In recent years, imputation has become a key tool in the success of genome-wide association studies. Genotype imputation has proven to increase the power of genetic association studies, by boosting the number of SNPs to be tested for association and facilitating the detection of rare variants in addition to common variants $[14,19,34,35]$. Furthermore, imputation aids in fine-mapping studies of the disease-associated region thus increasing the chance of identifying additional candidate SNPs [36]. Finally, genotype imputation enables metaanalysis that combines results across studies based on different genotyping platforms [37,38]. This approach has been effective in identifying novel associations in different traits [39-44]. 


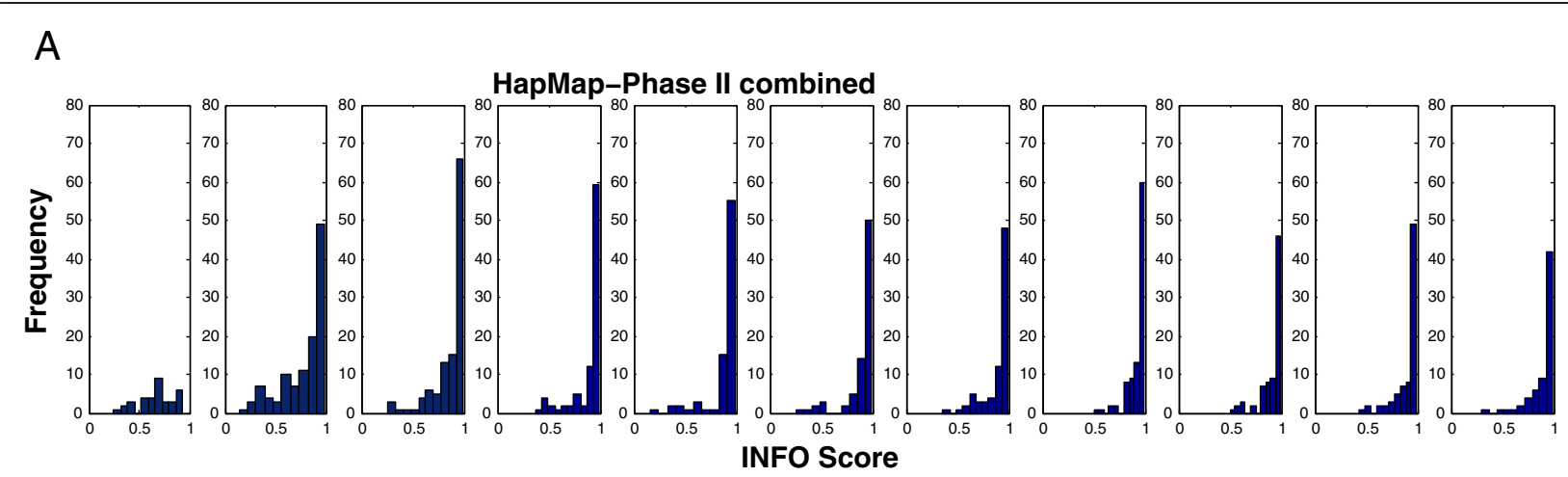

B
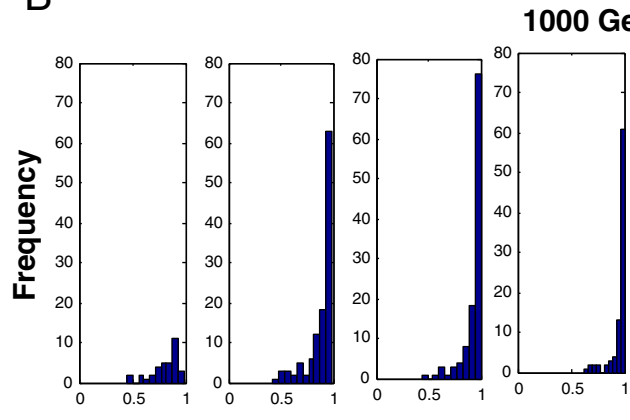

1000 Genomes-Phase I

Figure 3 Histogram showing the distribution of INFO scores within each allele frequency bin. For the two-step imputation method based on the HapMap phase II combined reference panel. For the two-step imputation method based on the 1000 Genomes phase I (June 2011 release) reference panel.

However, an important concern with respect to imputation lies in the selection of an appropriate reference panel. Most of the GWAS to date have been conducted in populations well represented by the available reference panels (e.g. European or East Asian populations), and used only one relevant reference population during the imputation process $[6,43,45-47]$. However, for populations that are phylogenetically distant from the samples present in the reference panels, the selection of a suitable reference panel for imputation becomes less clear. In this situation, differences in the pattern of LD between the study and reference populations may affect imputation accuracy. Different approaches have been suggested for this particular scenario. For example, Huang et al. [16] explored imputation accuracy in the samples of the HGDP-CEPH panel, which is a worldwide collection of individuals from different locations, using the HapMap II reference panels. The authors found that for most of the studied samples, mixtures from at least two HapMap reference samples maximized imputation accuracy [16]. Another study showed that using tag SNPs from all the HapMap reference populations combined captured common variation in African American, Latino and Hawaiian samples more effectively than tag SNPs obtained from the individual HapMap reference samples [48]. This 'cosmopolitan' approach to imputation, combining reference haplotypes from all the reference populations available, is the strategy currently recommended by the most widely used imputation packages, such as IMPUTE $[19,20]$ and MACH [21].

African American and Hispanic/Latino populations have unique challenges for imputation. These populations are the result of recent admixture between continental groups (primarily European, Native American and West African populations) and admixture proportions show substantial geographic variation [49-51]. Several studies have evaluated imputation performance in recently admixed populations. In a recent GWAS of coronary heart disease and its risk factors in a large African American sample [52], a high imputation concordance (95.6\%) was obtained when SNPs were imputed using a combined reference panel of haplotypes from the HapMap phase II CEU and YRI panels. In another study in African Americans [53], the highest imputation yield and coverage were attained using the two HapMap reference panels (CEU and YRI) separately and then merging the results. Another approach for imputation in African American populations has been recently suggested by Paşaniuc et al. (2011) [54]. This strategy, termed 'local ancestry aware imputation', uses local ancestry to guide 


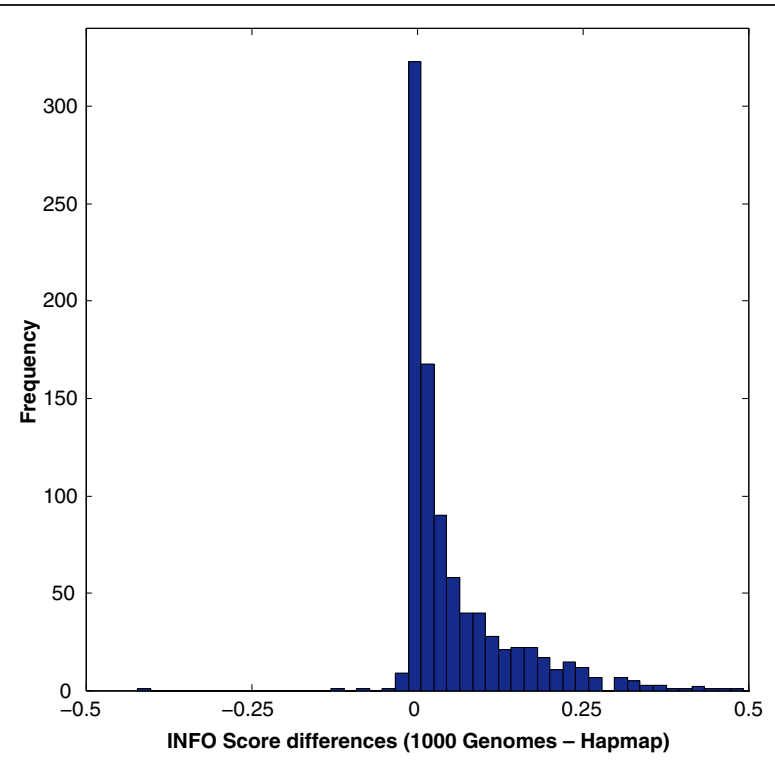

Figure 4 Histogram showing the distribution of the differences in the INFO scores of HapMap phase II combined and the 1000 Genomes phase I reference panels. The two-step imputation method based panels were only considered for this analysis.

the choice of reference haplotypes for imputation and shows marginal improvement in imputation accuracy in the admixed sample. However, this approach will be more difficult to implement in Hispanic/Latino populations, due to the lack of reference data for the relevant Native American parental populations, which is key to obtain accurate estimates of local ancestry. In the study by Huang et al. (2009), using combinations of two (European and East Asian) or three HapMap reference samples (East Asian, European and West African) produced the highest imputation accuracies (>95\%) for two Native American samples (Pima and Maya) and a sample from Colombia [16]. A recent study [55] showed that, when performing imputation in a Hispanic sample from San Francisco with the program IMPUTE

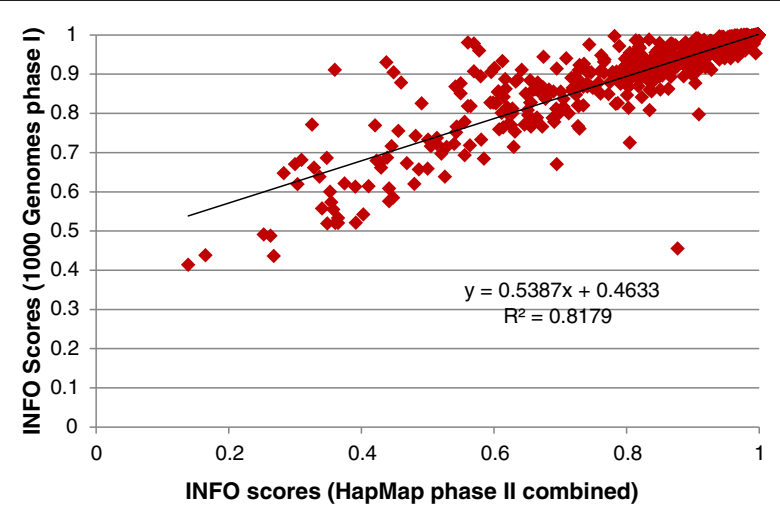

Figure 5 Correlation between the INFO scores for the two-step imputation method based on HapMap phase II combined and the 1000 Genomes phase I reference panels. v2 and the HapMap II reference panel, using local haplotype weights based on a coalescent method provided lower error rates $(7.8 \%)$ than using no weighting (8.9\%), or a global weighting method based on empirical estimates of ancestry (9.0\%) [56]. It is important to note that most of the aforementioned studies have used the HapMap II panel as the reference dataset for imputation. However, the recent progress of the 1000 Genomes project (http://www.1000genomes.org/) has provided the scientific community with much more complete reference panels, both in terms of the number of markers and the number of populations. Importantly, the reference databases are updated on a regular basis. For this reason, it is currently recommended to perform the imputation in two stages: pre-phasing the study genotypes to estimate haplotypes, and then imputing untyped genotypes in a separate run. This substantially reduces imputation time with respect to single-step approaches at the expense of a small loss in accuracy. An important advantage of this approach is that, as new reference data become available, it is only necessary to repeat the imputation step.

In this study, we evaluated the imputation performance of the widely used program IMPUTE in an admixed sample from Mexico City using different imputation strategies (single-step vs. two-step imputation) and reference panels (HapMap and 1000 Genomes). We have previously described that this sample primarily has Native American (62\%) and European contributions (33\%), with a low proportion of African ancestry (5\%) [30]. Importantly, there are no Native American reference samples in the HapMap or 1000 
Genomes datasets, so it is of relevance to test the relative imputation performance of these reference panels in the Mexican sample. In an analysis of imputed markers on chromosome 12, we observed that for this sample there are only minor differences in imputation accuracy between the single-step and two-step approaches (Table 1). The concordance rate of the single-step approach is only slightly higher than that of the two-step approach $(99.1 \%$ vs. $98.4 \%$ when using the HapMap phase II combined reference sample, respectively). In contrast, the imputation efficacy (i.e. proportion of non-missing genotypes) was higher for the twostep than the single-step imputation approach $(90.1 \%$ vs. $85.5 \%$, respectively). Therefore, our study confirms the two-step approach as the preferable imputation strategy, because it provides flexibility and faster imputation times, while providing an overly similar imputation performance to the single-step approach.

As expected, we observed that adding the HapMap Phase III Mexican American sample from LA to the HapMap Phase II combined reference sample there were marginal increases in both accuracy (99.4\% vs. 99.1\%) and efficacy (85.9\% vs. $85.5 \%)$ (Table 1$)$. We also anticipated to find that reducing the posterior probability thresholds when calling the imputed genotypes would result on lower imputation accuracy and higher proportions of non-missing genotypes. The reductions observed in imputation accuracy were relatively minor, from $98.4 \%$ with a posterior probability threshold of 0.9 to $95.5 \%$ with a threshold of 0.5 (Figure 1). This relatively small reduction in overall imputation accuracy is primarily due to the fact that most genotypes have very high posterior probabilities. Therefore, adding the relatively small percentage of genotypes with lower posterior probabilities (and lower concordance rates) does not produce a major shift in overall imputation accuracy. Of all masked markers on chromosome 12, 61.5\% had INFO scores higher than $0.9,15.4 \%$ had INFO scores between 0.8 and 0.9 , $6.5 \%$ had INFO scores between 0.7 and $0.8,6.2 \%$ had info scores between 0.6 and $0.7,3.8 \%$ had INFO scores between 0.5 and 0.6 , and $6.7 \%$ INFO scores lower than 0.5 (see also discussion below about the relationship of imputation efficacy and accuracy and allele frequency).

We also examined the potential improvement in imputation performance obtained with the recently available 1000 Genomes panel (June 2011 release), with respect to the HapMap panel, using the two-step imputation protocol. The 1000 Genomes panel is a much more comprehensive and powerful resource for imputation, comprising more than 37 million autosomal SNPs present in 1,094 individuals from different populations around the world. Here, we show that for the Mexican sample the major improvement associated with the use of the 1000 Genomes reference panel is the substantial increase in imputation efficacy, in addition to the larger number of imputed markers (Table 1). Genotype concordances were similar for both reference datasets (around 98.4\%). However, imputations with the 1000 Genomes panel resulted in $94.7 \%$ of non-missing genotypes (employing a posterior probabiity threshold of 0.9), in comparison with $90.1 \%$ for the HapMap phase II combined panel (using the same threshold). When the marker INFO scores are plotted for different allele frequency bins, either as an average (Figure 2) or as histograms of the individual scores (Figures $3 \mathrm{~A}$ and $3 \mathrm{~B}$ ), it is evident that the confidence of the genotype calls is higher with the 1000 Genomes panel for all allele frequency categories. There is a high correlation between the INFO scores obtained with the 1000 Genomes and HapMap phase II reference panels (Figure 5), but the former are systematically higher than the latter (Figure 4).

The results described above are based on an analysis of markers on chromosome 12. An analysis of markers on chromosome 22 gives consistent results: The concordance rates using the HapMap phase II and 1000 Genomes reference panels are very similar (97.6\% vs. $97.3 \%$, respectively), but the proportion of non-missing genotypes is lower with the HapMap reference panel than with the 1000 Genomes panel (83.2\%, and 89.9\%, respectively). Interestingly, in the HLA region on chromosome 6, which spans approximately 5 megabases (29$34 \mathrm{Mb}$ ) and has shown signatures of natural selection in previous studies (31-33), both the imputation accuracy (concordance) and the imputation efficacy (proportion of non-missing genotypes) were higher than those observed for chromosomes 12 and 22. When analyzing locus ancestry with a panel of Ancestry Informative Markers in the sample from Mexico City (data not shown), we observed that in a broad region of chromosome 6, including the HLA loci, there was an excess of European ancestry with respect to the rest of the genome, in both type 2 diabetes patients and controls. This may be a potential explanation for the increased imputation accuracy and efficacy identified in the HLA region (i.e. both reference panels, HapMap and 1000 Genomes, have a good representation of European populations, but Native American populations are not well represented in these panels).

The imputation performance of the 1000 Genomes reference panel for rare variants is substantially better than that of the HapMap phase II panel. However, the average imputation confidence (INFO score) is considerably lower for rare variants than for common variants (Figures 2 and 3), irrespective of the reference panel. The rare alleles $(<1 \%)$ present in the Mexican sample are not properly captured by any of the reference panels, in spite of the inclusion in the 1000 Genomes panel of dense data from another sample of Mexican ancestry from LA. 
This is also evident in a more detailed comparison of imputation accuracy and efficacy for heterozygotes in the following allele frequency categories: $<1 \%, 1-5 \%$ and $45-50 \%$. For common variants $(45-50 \%)$, the imputation accuracy and efficacy were very high ( $>97 \%$ concordance and $>90 \%$ non-missing genotypes). However, for rare variants $(<1 \%)$, the proportion of missing genotypes was quite high $(>21 \%)$, and importantly, even for the genotypes with high posterior probabilities $(>0.9)$, there was a large proportion of discordant calls $(>39 \%)$. It is important to note that our analyses were based on markers from a commercial microarray (in order to minimize genotyping errors, the program PLINK was used to merge the genotype calls obtained with two genotyping algorithms: BRLMM-P and Birdseed), and it is not clear to which extent these findings can be extrapolated to other scenarios (e.g. sequencing data). However, our results highlight the need to be cautious with the interpretation of the results for rare variants in GWAS in Hispanic samples.

\section{Conclusions}

We show that the program IMPUTE has an excellent imputation performance for common markers in an admixed sample from Mexico City, which has primarily Native American (62\%) and European (33\%) contributions. Genotype concordances for randomly masked markers are higher than $98.4 \%$ using different imputation strategies, in spite of the fact that no Native American samples are present in the HapMap and 1000 Genomes reference panels. In this sample, the best balance of imputation accuracy and efficiency was obtained with the 1,000 Genomes panel (genotype concordance 98.4\% and proportion of non-missing genotypes $94.7 \%$ ). However, not unexpectedly, rare variants (frequencies $<1 \%$ ) are not captured efficiently by any of the available panels.

\section{Competing interests}

The authors declare that they have no competing interests.

\section{Authors' contributions}

SK carried out the imputations with the program IMPUTE, analyzed the imputation results and wrote the manuscript. AVS, JP, JEP, JKR and MC recruited participants in Mexico City and coordinated the extraction of DNA. EJP coordinated the genotyping of the samples, conceived the study, and helped to draft the manuscript. All authors read and approved the final manuscript

\section{Acknowledgements}

We thank all the individuals who participated in the study. In Canada this work was supported by the Canadian Institutes of Health Research (Operating Grant and Early Investigator Award to EJP), the Banting and Best Diabetes Centre (New Investigator Award to EJP and Postdoctoral Fellowship to SK), the Canada Foundation for Innovation and the Ontario Innovation Trust (EJP). In Mexico this research was supported by CONACYT SALUD-2005C02-14412, Proyectos Estrategicos, Apoyo Financiero Fundacion IMSS and Fundacion Gonzalo Rio Arronte. MC is a recipient of a Fundacion IMSS Scholarship, Mexico. We would like to acknowledge Dr. Shovonlal Roy for helping us in generating the figures.

\section{Author details}

'Department of Anthropology, University of Toronto at Mississauga, 3359 Mississauga Road North, Mississauga, ON, Canada. ${ }^{2}$ Unidad de Investigacion Medica en Bioquimica, Hospital de Especialidades, Centro Medico Nacional Siglo XXI, IMSS, Av. Cuauhtemoc 330, Col. DoctoresC.P. 06720, Mexico City, Mexico. ${ }^{3}$ Unidad de Investigacion en Epidemiologia Clinica, Hospital General Regional 1, Dr Carlos McGregor, IMSS, Mexico City, Mexico. ${ }^{4}$ Fundacion IMSS, Mexico City, Mexico.

Received: 2 December 2011 Accepted: 5 April 2012

Published: 1 May 2012

\section{References}

1. Kruglyak $L$ : Prospects for whole-genome linkage disequilibrium mapping of common disease genes. Nat Genet 1999, 22:139-144.

2. Sebastiani P, Timofeev N, Dworkis DA, Perls TT, Steinberg MH: Genomewide association studies and the genetic dissection of complex traits. Am $J$ Hematol 2009, 84:504-515.

3. Kruglyak L: The road to genome-wide association studies. Nat Rev Genet 2008, 9:314-318

4. McCarthy MI, Abecasis GR, Cardon LR, Goldstein DB, Little J, loannidis JP, Hirschhorn JN: Genome-wide association studies for complex traits: consensus, uncertainty and challenges. Nat Rev Genet 2008, 9:356-369.

5. Frazer KA, Murray SS, Schork NJ, Topol EJ: Human genetic variation and its contribution to complex traits. Nat Rev Genet 2009, 10:241-251.

6. The Wellcome Trust Case Control Consortium: Genome-wide association study of 14,000 cases of seven common diseases and 3,000 shared controls. Nature 2007, 447:661-678.

7. Tsuchihashi Z, Dracopoli NC: Progress in high throughput SNP genotyping methods. Pharmacogenomics J 2002, 2:103-110.

8. Low YL, Wedrén S, Liu J: High-throughput genomic technology in research and clinical management of breast cancer. Evolving landscape of genetic epidemiological studies. Breast Cancer Res 2006, 8:209.

9. Ku CS, Kasiman K, Chia KS: High-Throughput Single Nucleotide Polymorphisms Genotyping Technologies. In: Encyclopedia of Life Sciences (ELS). Edited by John Wiley \& Sons, Ltd. Chichester, 2009, http://www.els.net [doi: 10.1002/9780470015902.a0021631].

10. Wang WY, Barratt BJ, Clayton DG, Todd JA: Genome-wide association studies: theoretical and practical concerns. Nat Rev Genet 2005, 6:109-118.

11. Barrett JC, Cardon LR: Evaluating coverage of genome-wide association studies. Nat Genet 2006, 38:659-662.

12. Pe'er I, de Bakker PI, Maller J, Yelensky R, Altshuler D, Daly MJ: Evaluating and improving power in whole-genome association studies using fixed marker sets. Nat Genet 2006, 38:663-667.

13. Donnelly P: Progress and challenges in genome-wide association studies in humans. Nature 2008, 456:728-731.

14. Marchini J, Howie B: Genotype imputation for genome-wide association studies. Nat Rev Genet 2010, 11:499-511.

15. Li Y, Willer C, Sanna S, Abecasis G: Genotype Imputation. Annu Rev Genomics Hum Genet 2009, 10:387-406.

16. Huang L, Li Y, Singleton AB, Hardy JA, Abecasis G, Rosenberg NA, Scheet P: Genotype-imputation accuracy across worldwide human populations. Am J Hum Genet 2009, 84(2):235-250

17. Anderson CA, Pettersson FH, Barrett JC: Evaluating the effects of imputation on the power, coverage, and cost efficiency of genome-wide SNP platforms. Am J Hum Genet 2008, 83:112-119.

18. Almeida MA, Oliveira PS, Pereira TV, Krieger JE, Pereira AC: An empirical evaluation of imputation accuracy for association statistics reveals increased type-l error rates in genome-wide associations. BMC Genet 2011, 12:10.

19. Marchini J, Howie B, Myers S, McVean G, Donnelly P: A new multipoint method for genome-wide association studies by imputation of genotypes. Nat Genet 2007, 39:906-913.

20. Howie BN, Donnelly P, Marchini J: A flexible and accurate genotype imputation method for the next generation of genome-wide association studies. Plos Genet 2009, 5(6):e1000529.

21. Li Y, Willer CJ, Ding J, Scheet P, Abecasis GR: MaCH: using sequence and genotype data to estimate haplotypes and unobserved genotypes. Genet Epidemiol 2010, 34:816-834.

22. Browning SR, Browning BL: Rapid and accurate haplotype phasing and missing-data inference for whole-genome association studies by use of 
localized haplotype clustering. Am J Hum Genet 2007, 81:1084-1097.

23. Scheet $P$, Stephens $M: A$ fast and flexible statistical model for large-scale population genotype data: applications to inferring missing genotypes and haplotypic phase. Am J Hum Genet 2006, 78:629-644.

24. Purcell S, Neale B, Todd-Brown K, Thomas L, Ferreira MA, Bender D, Maller J, Sklar P, de Bakker PI, Daly MJ, Sham PC: PLINK: a tool set for wholegenome association and population-based linkage analyses. Am J Hum Genet 2007, 81:559-575.

25. Pei YF, Li J, Zhang L, Papasian CJ, Deng HW: Analyses and comparison of accuracy of different genotype imputation methods. PLoS One 2008, 3 (10):e3551.

26. Browning SR: Missing data imputation and haplotype phase inference for genome-wide association studies. Hum Genet 2008, 124(5):439-450.

27. Nothnagel M, Ellinghaus D, Schreiber S, Krawczak M, Franke A: A comprehensive evaluation of SNP genotype imputation. Hum Genet 2009, 125(2):163-171.

28. Hao K, Chudin E, McElwee J, Schadt EE: Accuracy of genome-wide imputation of untyped markers and impacts on statistical power for association studies. BMC Genet 2009, 10:27.

29. Pei YF, Zhang L, Li J, Deng HW: Analyses and comparison of imputationbased association methods. PLoS One 2010, 5(5):e10827.

30. Parra EJ, Below JE, Krithika S, Valladares A, Barta JL, Cox NJ, Hanis CL, Wacher N, Garcia-Mena J, Hu P, Shriver MD: Diabetes Genetics Replication and Meta-analysis (DIAGRAM) Consortium, Kumate J, McKeigue PM, Escobedo J, Cruz M: Genome-wide association study of type 2 diabetes in a sample from Mexico City and a meta-analysis of a MexicanAmerican sample from Starr County, Texas. Diabetologia 2011, 54(8): 2038-2046.

31. Bhatia G, Patterson N, Pasaniuc B, Zaitlen N, Genovese G, Pollack S, Mallick S, Myers S, Tandon A, Spencer C, Palmer CD, Adeyemo AA, Akylbekova EL, Cupples LA, Divers J, Fornage M, Kao WH, Lange L, Li M, Musani S, Mychaleckyj JC, Ogunniyi A, Papanicolaou G, Rotimi CN, Rotter Jl, Ruczinski I, Salako B, Siscovick DS, Tayo BO, Yang Q, et al: Genome-wide comparison of African-ancestry populations from CARe and other cohorts reveals signals of natural selection. Am J Hum Genet 2011, 89(3):368-381.

32. Buhler S, Sanchez-Mazas A: HLA DNA sequence variation among human populations: molecular signatures of demographic and selective events. PLoS One 2011, 6(2):e14643.

33. Albrechtsen A, Moltke I, Nielsen R: Natural selection and the distribution of identity-by-descent in the human genome. Genetics 2010, 186(1):295-308.

34. Guan Y, Stephens M: Practical issues in imputation-based association mapping. PLoS Genet 2008, 4(12):e1000279.

35. Spencer CC, Su Z, Donnelly P, Marchini J: Designing genome-wide association studies: sample size, power, imputation, and the choice of genotyping chip. PLoS Genet 2009, 5(5):e1000477.

36. Liu JZ, Tozzi F, Waterworth DM, Pillai SG, Muglia P, Middleton L, Berrettini W, Knouff CW, Yuan X, Waeber G, Vollenweider P, Preisig M, Wareham NJ, Zhao JH, Loos RJ, Barroso I, Khaw KT, Grundy S, Barter P, Mahley R, Kesaniemi A, McPherson R, Vincent JB, Strauss J, Kennedy JL, Farmer A, McGuffin P, Day R, Matthews K, Bakke P, et al: Wellcome Trust Case Control Consortium, Mooser V, Francks C, Marchini J: Meta-analysis and imputation refines the association of $15 q 25$ with smoking quantity. Nat Genet 2010, 42 (5):436-40.

37. de Bakker PI, Ferreira MA, Jia X, Neale BM, Raychaudhuri S, Voight BF: Practical aspects of imputation-driven meta-analysis of genome-wide association studies. Hum Mol Genet 2008, 17(R2):R122-128.

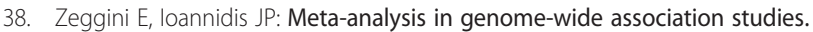
Pharmacogenomics 2009, 10(2):191-201.

39. Cooper JD, Smyth DJ, Smiles AM, Plagnol V, Walker NM, Allen JE, Downes $K$, Barrett JC, Healy BC, Mychaleckyj JC, Warram JH, Todd JA: Meta-analysis of genome-wide association study data identifies additional type 1 diabetes risk loci. Nat Genet 2008, 40(12):1399-1401.

40. De Jager PL, Jia X, Wang J, de Bakker PI, Ottoboni L, Aggarwal NT, Piccio L, Raychaudhuri S, Tran D, Aubin C, Briskin R, Romano S, International MS Genetics Consortium, Baranzini SE, McCauley JL, Pericak-Vance MA, Haines JL, Gibson RA, Naeglin Y, Uitdehaag B, Matthews PM, Kappos L, Polman C, McArdle WL, Strachan DP, Evans D, Cross AH, Daly MJ, Compston A, Sawcer $\mathrm{SJ}$, et al: Meta-analysis of genome scans and replication identify CD6, IRF8 and TNFRSF1A as new multiple sclerosis susceptibility loci. Nat Genet 2009, 41(7):776-782.
41. Houlston RS, Cheadle J, Dobbins SE, Tenesa A, Jones AM, Howarth K, Spain SL, Broderick P, Domingo E, Farrington S, Prendergast JG, Pittman AM, Theodoratou E, Smith CG, Olver B, Walther A, Barnetson RA, Churchman M, Jaeger EE, Penegar S, Barclay E, Martin L, Gorman M, Mager R, Johnstone E, Midgley R, Niittymäki I, Tuupanen S, Colley J, Idziaszczyk S, et al: Metaanalysis of three genome-wide association studies identifies susceptibility loci for colorectal cancer at 1q41, 3q26.2, 12q13.13 and 20q13.33. Nat Genet 2010, 42(11):973-977.

42. International Parkinson Disease Genomics Consortium, Nalls MA, Plagnol V, Hernandez DG, Sharma M, Sheerin UM, Saad M, Simón-Sánchez J, Schulte C, Lesage S, Sveinbjörnsdóttir S, Stefánsson K, Martinez M, Hardy J, Heutink P, Brice A, Gasser T, Singleton AB, Wood NW: Imputation of sequence variants for identification of genetic risks for Parkinson's disease: a metaanalysis of genome-wide association studies. Lancet 2011, 377(9766): 641-649.

43. Strawbridge RJ, Dupuis J, Prokopenko I, Barker A, Ahlqvist E, Rybin D, Petrie JR, Travers ME, Bouatia-Naji N, Dimas AS, Nica A, Wheeler E, Chen H, Voight BF, Taneera J, Kanoni S, Peden JF, Turrini F, Gustafsson S, Zabena C, Almgren P, Barker DJ, Barnes D, Dennison EM, Eriksson JG, Eriksson P, Eury E, Folkersen L, Fox CS, Frayling TM, et al: Genome-wide association identifies nine common variants associated with fasting proinsulin levels and provides new insights into the pathophysiology of type 2 diabetes. Diabetes 2011, 60(10):2624-2634

44. Zeggini E, Scott $\sqcup$, Saxena R, Voight BF, Marchini JL, Hu T, de Bakker PI, Abecasis GR, Almgren P, Andersen G, Ardlie K, Boström KB, Bergman RN, Bonnycastle LL, Borch-Johnsen K, Burtt NP, Chen H, Chines PS, Daly MJ, Deodhar P, Ding CJ, Doney AS, Duren WL, Elliott KS, Erdos MR, Frayling TM, Freathy RM, Gianniny L, Grallert H, Grarup N, et al: Meta-analysis of genomewide association data and large-scale replication identifies additional susceptibility loci for type 2 diabetes. Nat Genet 2008, 40(5):638-645.

45. Scott L, Mohlke KL, Bonnycastle LL, Willer CJ, Li Y, Duren WL, Erdos MR, Stringham HM, Chines PS, Jackson AU, Prokunina-Olsson L, Ding CJ, Swift AJ, Narisu N, Hu T, Pruim R, Xiao R, Li XY, Conneely KN, Riebow NL, Sprau AG, Tong M, White PP, Hetrick KN, Barnhart MW, Bark CW, Goldstein JL, Watkins $L$, Xiang F, Saramies J, et al: A genome-wide association study of type 2 diabetes in Finns detects multiple susceptibility variants. Science 2007, 316(5829):1341-1345.

46. Kato N, Takeuchi F, Tabara Y, Kelly TN, Go MJ, Sim X, Tay WT, Chen CH, Zhang Y, Yamamoto K, Katsuya T, Yokota M, Kim YJ, Ong RT, Nabika T, Gu D, Chang LC, Kokubo Y, Huang W, Ohnaka K, Yamori Y, Nakashima E, Jaquish CE, Lee JY, Seielstad M, Isono M, Hixson JE, Chen YT, Miki T, Zhou X, et al: Meta-analysis of genome-wide association studies identifies common variants associated with blood pressure variation in east Asians. Nat Genet 2011, 43(6):531-8.

47. Kim YJ, Go MJ, Hu C, Hong CB, Kim YK, Lee JY, Hwang JY, Oh JH, Kim DJ, Kim NH, Kim S, Hong EJ, Kim JH, Min H, Kim Y, Zhang R, Jia W, Okada Y, Takahashi A, Kubo M, Tanaka T, Kamatani N, Matsuda K, Consortium MAGIC, Park T, Oh B, Kimm K, Kang D, Shin C, Cho NH, et al: Large-scale genomewide association studies in East Asians identify new genetic loci influencing metabolic traits. Nat Genet 2011, 43(10):990-995.

48. de Bakker PI, Burtt NP, Graham RR, Guiducci C, Yelensky R, Drake JA, Bersaglieri T, Penney KL, Butler J, Young S, Onofrio RC, Lyon HN, Stram DO, Haiman CA, Freedman ML, Zhu X, Cooper R, Groop L, Kolonel LN, Henderson BE, Daly MJ, Hirschhorn JN, Altshuler D: Transferability of tag SNPs in genetic association studies in multiple populations. Nat Genet 2006, 38(11):1298-1303.

49. Parra EJ, Marcini A, Akey J, Martinson J, Batzer MA, Cooper R, Forrester T, Allison DB, Deka R, Ferrell RE, Shriver MD: Estimating African American admixture proportions by use of population-specific alleles. Am J Hum Genet 1998, 63(6):1839-1851.

50. Wang S, Ray N, Rojas W, Parra MV, Bedoya G, Gallo C, Poletti G, Mazzotti G, Hill K, Hurtado AM, Camrena B, Nicolini H, Klitz W, Barrantes R, Molina JA, Freimer NB, Bortolini MC, Salzano FM, Petzl-Erler ML, Tsuneto LT, Dipierri JE, Alfaro EL, Bailliet G, Bianchi NO, Llop E, Rothhammer F, Excoffier L, RuizLinares A: Geographic patterns of genome admixture in Latin American Mestizos. PLoS Genet 2008, 4(3):e1000037.

51. Galanter JM, Fernandez-Lopez JC, Gignoux CR, Barnholtz-Sloan J, FernandezRozadilla C, Via M, Hidalgo-Miranda A, Contreras AV, Figueroa LU, Raska P, Jimenez-Sanchez G, Silva Zolezzi I, Torres M, Ponte CR, Ruiz Y, Salas A, Nguyen E, Eng C, Borjas L, Zabala W, Barreto G, Rondón González F, Ibarra A, 
Taboada P, Porras L, Moreno F, Bigham A, Gutierrez G, Brutsaert T, LeónVelarde F, et al: for the LACE Consortium. Development of a Panel of Genome-Wide Ancestry Informative Markers to Study Admixture Throughout the Americas. PLoS Genet 2012, 8(3):1002554.

52. Lettre $G$, Palmer $C D$, Young $T$, Ejebe $K G$, Allayee $H$, Benjamin EJ, Bennett $F$, Bowden DW, Chakravarti A, Dreisbach A, Farlow DN, Folsom AR, Fornage M, Forrester T, Fox E, Haiman CA, Hartiala J, Harris TB, Hazen SL, Heckbert SR, Henderson BE, Hirschhorn JN, Keating BJ, Kritchevsky SB, Larkin E, Li M, Rudock ME, McKenzie CA, Meigs JB, Meng YA, et al: Genome-wide association study of coronary heart disease and its risk factors in 8,090 African Americans: the NHLBI CARe Project. PLoS Genet 2011, 7(2): e1001300.

53. Shriner D, Adeyemo A, Chen G, Rotimi CN: Practical considerations for imputation of untyped markers in admixed populations. Genet Epidemiol 2010, 34(3):258-265.

54. Paşaniuc B, Zaitlen N, Lettre G, Chen GK, Tandon A, Kao WH, Ruczinski I, Fornage M, Siscovick DS, Zhu X, Larkin E, Lange LA, Cupples LA, Yang O Akylbekova EL, Musani SK, Divers J, Mychaleckyj J, Li M, Papanicolaou GJ, Millikan RC, Ambrosone CB, John EM, Bernstein L, Zheng W, Hu JJ, Ziegler RG, Nyante SJ, Bandera EV, Ingles SA, et al: Enhanced statistical tests for GWAS in admixed populations: assessment using African Americans from CARe and a Breast Cancer Consortium. PLoS Genet 2011, 7(4): e1001371.

55. Paşaniuc B, Avinery R, Gur T, Skibola CF, Bracci PM, Halperin E: A generic coalescent-based framework for the selection of a reference panel for imputation. Genet Epidemiol 2010, 34(8):773-782.

56. Egyud MR, Gajdos ZK, Butler JL, Tischfield S, Le Marchand L, Kolonel LN, Haiman CA, Henderson BE, Hirschhorn JN: Use of weighted reference panels based on empirical estimates of ancestry for capturing untyped variation. Hum Genet 2009, 125(3):295-303.

doi:10.1186/1755-8794-5-12

Cite this article as: Krithika et al: Evaluation of the imputation

performance of the program IMPUTE in an admixed sample from Mexico City using several model designs. BMC Medical Genomics 2012 $5: 12$

\section{Submit your next manuscript to BioMed Central and take full advantage of:}

- Convenient online submission

- Thorough peer review

- No space constraints or color figure charges

- Immediate publication on acceptance

- Inclusion in PubMed, CAS, Scopus and Google Scholar

- Research which is freely available for redistribution 